\title{
Classroom Intelligent Lighting Control System Based on 51 Single - chip Microcomputer
}

\author{
Hong-Lai Yan
}

School of Applied Technology, Xi'an International University, Xi'an, Shaanxi, PR. China 710077

Keywords: 51 Single Chip Microcomputer; Lighting Control

\begin{abstract}
University of traditional lighting control are ever burning lights, no lights "phenomenon, in order to solve the traditional university classrooms for energy waste phenomenon, specially research the classroom lights use methods and existing disadvantages. This paper uses a simple 51 micro controller based control circuit, coupled with a number of auxiliary circuit modules, to achieve the development and testing of the test system. Including data acquisition module, serial communication module and the implementation of the module circuit design and software development.
\end{abstract}

\section{Introduction}

In nowadays, the world's major problem is still a waste of energy or how to develop a new energy, especially in the late 20th century, many scientists put forward to the idea that our country's policy is energy-saving emission reduction, the energy issue Pushed to the number of problem to solve. Against the background of the above, can deeply feel the school's waste of electricity is particularly serious, especially in many classroom lighting without class, which nobody study hall, but it has been on the lights. So for the kind of building before the cover, this phenomenon is very serious, so wehave toi against this situation. I will study how to solve this problem. I use this article is a simple 51 single-chip microcomputer as the main control circuit, and interspersed with some of the auxiliary circuit module to achieve the test system development and debugging. Including data acquisition module, serial communication module and the implementation of the circuit design and software development.

\section{Basic Design Ideas}

For this intelligent lighting system, divided into hardware and software parts. The hardware part mainly uses 51 monolithic integrated circuits as the core component, the main reason is the 51 monolithic integrated circuit price-performance ratio is also high and the stability. The other are some auxiliary modules, such as the clock module, infrared tube module. Software is the main part of the circuit diagram with the simulation, the main use of the $\mathrm{C}$ language programming, debugging process plus simulation. And puts forward the concrete realization method for the control strategy of the system. And the use of $\mathrm{C}$ language to write programs, and then use the relevant software simulation. The main core of the system design is the choice of the controller.

Option 1: Use the 51 Series as the system controller

AT89C51 as a system control program. Because of its low power consumption, small size, mature technology and low cost, it is widely used in various fields. Because of the advantages of the chip, it can be used in a wide range of applications. Feet less, in the hardware is very easy to achieve. And anti-jamming performance.

Option 2: The MSP43OF149 series microcontroller for the system controller.MSP430F149 series MCU can achieve a variety of complex logic functions, large modules, high density, it will all devices integrated in a chip, reducing the size and improve stability. 
Because MSP430F149 series price is much more expensive than 51 single-chip computers, and this design can deal with the speed requirement in general, do not need too high processing speed, integrated design performance and benefits, this design chooses scheme 1 .

\section{System Hardware Design}

STC89C51 Microcontroller. The STC89C51 is a low-voltage, high-performance CMOS 8-bit micro controller with a $4 \mathrm{~K}$ byte operating voltage range of $3.4 \mathrm{~V}$ to $5.5 \mathrm{~V}$ and operating frequency range of 0 to $35 \mathrm{MHz}$ with two 16-bit counters. In addition STC89C51 single-chip download process in the process, if there is power failure, download failure tips, STC89C51 single-chip inside the original download the original data is retained in the inside, still can run on the card slot, if other program data, You have to re-download. The maximum operating frequency of $35 \mathrm{Mhz}, 6 \mathrm{~T} / 12 \mathrm{~T}$ optional.

Reset Circuit. Reset circuit is mainly a device to restore the circuit, and its major function with the calculator quite, the difference is that the start of this one, the principle there are different methods. Calculator is mainly press the "OFF" button to achieve clear, but the reset circuit is not the same, the first is to restore the state before the circuit, and then one is to have to operate their own, there is no automatic, and finally if you want to Automatic words must be written procedures in order to achieve the automatic realization of the reset function.

Display Module Circuit. In this system design that is the main part of the LED digital tube, this is the display link, a LED digital tube can only appear a number, but others can display letters. Otherwise like some relatively similar letters is very difficult to distinguish between the LED display, the resolution is still relatively low, the best display method or display the number. The main advantage is that the price is acceptable, the cost to a minimum. As shown in Figure 1

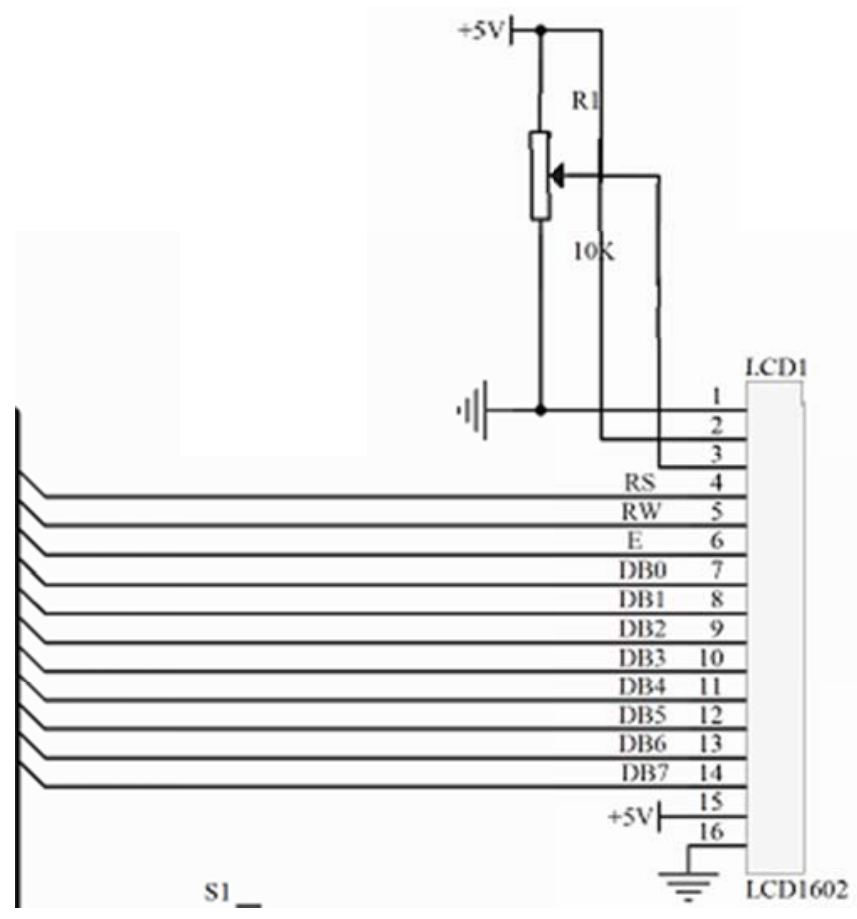

Figure1 The main advantage

Infrared Tube Control Circuit. The infrared tube on the main core component is the PN junction, the components of the major components of the semiconductor components are combined together to achieve this function. Infrared receiver diode in the general illumination of light irradiation, the resulting current called photocurrent. If the external circuit connected to the load, the 
load to obtain the electrical signal, and the electrical signal changes with the corresponding changes in light. As shown in Figure 2

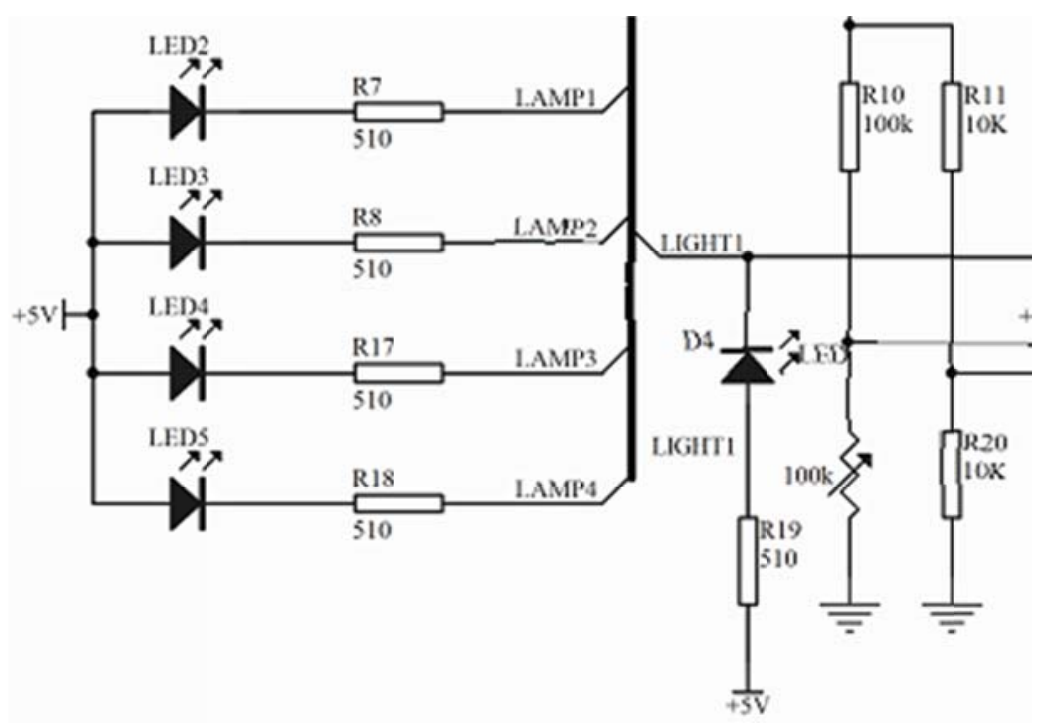

Figure 2 the load to obtain the electrical signal, and the electrical signal changes with the corresponding changes in light

Clock Circuit. Clock circuit is able to produce a certain frequency of the standard clock crystal oscillator circuit. No matter what time period or time point, they are in accordance with the corresponding smooth work. The work of this process is clock circuit. Now I can collect information through the Internet can see basically using the chip as a timer DS1302, the chip model is Dallas production company, it has one of the greatest benefits is =has a strong charge capacity, characterized by string Line way to transfer data between, but also has the function of charging the power supply. The general situation is to use $37.235 \mathrm{KHZ}$ crystal. The diagram shown in Figure 3 below.

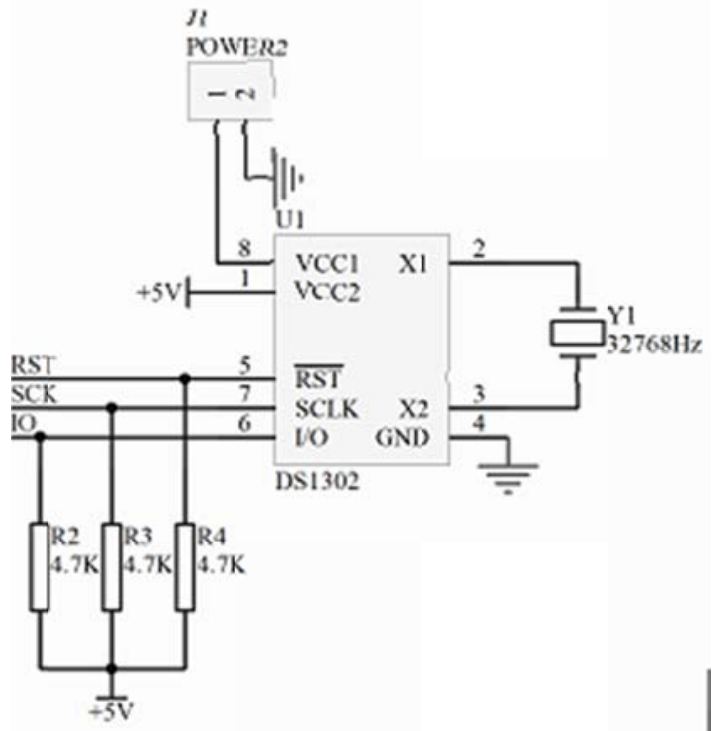

Figure 3 The general situation is to use $37.235 \mathrm{KHZ}$ crystal

\section{System Software Design}

This article has one simulation flow chart, the first is the main flow chart, shown in Figure 4: 


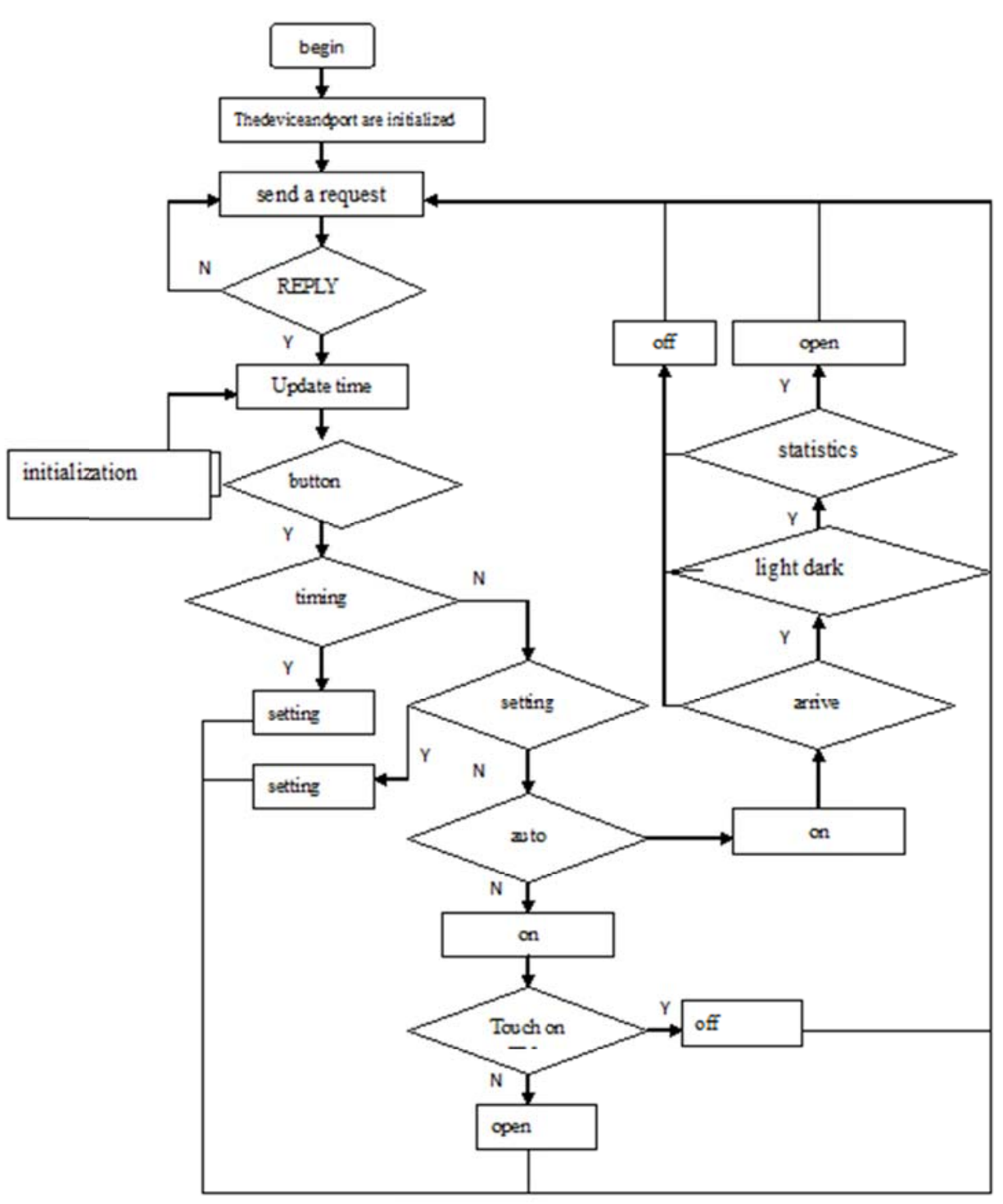

Figure 4 simulation flow chart

\section{Debugging and Implementation}

Operation consists of two parts, the first is to set the time, the second is to set the timing of the start time and end time, the third is the mode switch and manual switch.

Under normal circumstances, real-time display, when the need for key operations, according to the following steps: Long press the Select button once after the release, shown in Figure 5.

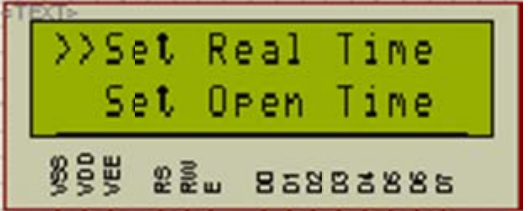

Figure 5 Long press the Select button once after the release

And then press the Enter key to enter the real-time settings. Press the Select button twice, as shown in Figure 6 


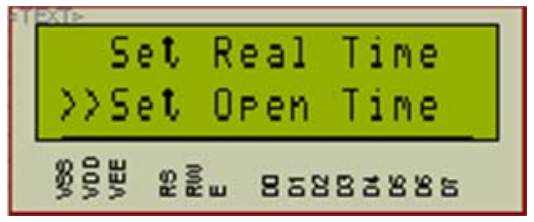

Figure 6 then press the Enter key to enter the real-time settings And then press the OK button to enter the set time.

\section{Conclusion}

Through the above methods, the content is based on 51 single-chip street lighting system design. This article uses a simple 51 single-chip microcomputer as the main control circuit, and interspersed with some auxiliary circuit module, to achieve the test system development and debugging. Including data acquisition module, serial communication module and the implementation of the circuit design and software development.

\section{References}

[1] Wang Jingxia. Application Technology of Single Chip Microcomputer. Beijing: Electronic Industry Press

[2] Chen $\mathrm{Zi}$. Practical Electronic Circuit Design and Debugging. Beijing: China Electric Power Press, 2014.6

[3] Zhao negative map. Sensor Integrated Circuit Manual. First Edition: Chemical Industry Press

[4] Tang Haoqiang. C programming tutorial. ISBN 978-7-302-15157-9: Tsinghua University Press

[5] Zhao Peihua. Principle and Interface Technology of Single Chip Microcomputer. Beijing: Mechanical Industry Press 